\title{
A Toolset for Simulink Improving Software Engineering Practices in Development with Simulink
}

\author{
Vera Pantelic, Steven Postma, Mark Lawford, Alexandre Korobkine, Bennett Mackenzie, Jeff Ong \\ and Marc Bender \\ McMaster Centre for Software Certification, Department of Computing and Software, \\ McMaster University, Hamilton, ON, Canada \\ pantelv@mcmaster.ca, steven.m.postma@gmail.com, \{lawford,korobka, mackeb,ongjf2, bendermm\}@mcmaster.ca
}

\begin{abstract}
Keywords: $\quad$ Simulink, Tools, Software Engineering, Transformation, Data Flow.
Abstract: This paper presents a set of tools that provide automatic support for application of some of the traditional software engineering practices when developing with Simulink. The tools are the: Signature Tool, Reach/Coreach Tool, Data Store Push-Down Tool, and Auto Layout Tool. The Signature Tool extracts the interface of a Simulink subsystem, identifying the subsystem's explicit, and implicit data flow mechanisms, empowering developers to use the implicit mechanisms more effectively. The Reach/Coreach Tool identifies data and control flow dependencies in a Simulink model and uses the information for model slicing. The view of dependencies offered by the tool significantly eases the comprehension of large models. The dependencies can also serve as indicators of alternative designs, and facilitate more effective testing and verification. The Data Store Push-Down Tool restricts the scope of Simulink's data stores thereby providing improved encapsulation, and increasing modularity. Finally, the Auto Layout Tool significantly decreases the manual effort developers spend in achieving proper layout of models during design and refactoring, and can be used by automated refactoring and transformation tools.
\end{abstract}

\section{INTRODUCTION}

The use of MATLAB/Simulink as a model-based design environment is wide spread across industries. While there are a number of reasons for its popularity (e.g., automatic code generation, rich modeling and simulation capabilities, existence of a large number of MathWorks and third party tools to aid development within the environment), the environment still lacks proper support for the application of some traditional software engineering practices. For example, Simulink does not have facilities to provide for data stores $^{1}$ to be declared read-only. Further, Simulink lacks self-documenting capabilities of imperative programming languages: for example, an analogue of a module interface in $\mathrm{C}$, as defined in $\mathrm{C}$ header files, does not exist in Simulink.

The main goal of this paper is to enhance modelbased design with Simulink through the application of some well-known software engineering principles. Proper automatic support is crucial for integration of these practices in design with Simulink. Therefore,

\footnotetext{
${ }^{1}$ Data stores in Simulink are analogous to variables in traditional programming languages.
}

the toolset to be presented in this paper helps a developer apply some well-known software engineering principles in an automated manner, allowing for the proposed principles to be seamlessly integrated into an existing Simulink model-based software development process. We introduce four tools, namely: the Signature Tool, the Reach/Coreach Tool, the Data Store Push-Down Tool, and the Auto Layout Tool.

The Signature Tool extracts a Simulink subsystem's signature: a representation of the subsystem's interface. The concept of signatures was introduced in (Bender et al., 2014a; Bender et al., 2014b). Signatures improve subsystems' comprehensibility, and identify implicit data flow mechanisms in Simulink enabling their more effective use. Further, analysis of signatures can indicate the quality of modularization of a Simulink design. While the core capabilities of the tool were presented in (Bender et al., 2014a; Bender et al., 2014b), the current paper presents the tool in more detail, providing natural language requirements for the tool, rather than focusing on the formal definition of signatures as in (Bender et al., 2014a; Bender et al., 2014b). Further, signatures have previously only been defined for virtual subsystems, while 
in the current paper the Signature Tool is extended to support the extraction of signatures of nonvirtual subsystems. The tool has been used by our automotive industrial partner to automatically help document subsystems' interfaces as part of an application's software design description.

The Reach/Coreach Tool identifies, for specified Simulink blocks, parts of the model that depend on the specified blocks (Reach functionality), or parts of the model that the specified blocks depend upon (Coreach functionality). Both data flow and control dependencies are accounted for. After the dependencies have been identified, the extraneous blocks can be removed: therefore, the tool provides for model slicing. To the best of our knowledge, there exists only one comprehensive slicing technique for Simulink models (Reicherdt and Glesner, 2012), that takes into account both data and control dependencies. In (Reicherdt and Glesner, 2012), data dependencies are calculated by traversing signal lines, and control dependencies are derived from the calculation of Conditional Execution Contexts - Simulink schedules for execution of blocks modeling conditional dependence. Compared to the model slicing approach of (Reicherdt and Glesner, 2012), our tool accounts for not only the data flow through signal lines, but also the data flow through Simulink's data stores and From/Goto blocks, and provides finegrained data flow tracking through Simulink buses. The Reach/Coreach dependencies extracted by the tool significantly ease the comprehension of models, and provide for automatic impact analysis, significantly simplifying models' verification and testing.

Data stores in Simulink are analogous to variables in traditional programming languages. The Data Store Push-Down Tool identifies the data stores that have scopes larger than necessary; for each identified data store, its declaration is then pushed down the model hierarchy to the lowest level possible such that all the references to the data store are still within the data store's scope. The push-down operation improves modularity of Simulink designs. The tool was introduced in (Bender et al., 2014b). However, its application in (Bender et al., 2014b) was to merely illustrate the effectiveness of a software complexity metric introduced in that paper. In the current paper, however, the tool's potential applications in a modelbased software development process are discussed in detail, and its implementation is explained.

The last tool to be presented in this paper is not focused on the application of software engineering principles per se, but rather on relieving a major effort in developing with Simulink: achieving a proper layout of a model. Surprisingly, there does not exist a comprehensive commercial automatic layout tool for Simulink models. While novel Simulink automatic layout algorithms have been proposed (e.g., (Klauske and Dziobek, 2010; Klauske et al., 2012)), no tools based on the algorithms are available for download. Our approach reuses a graph drawing algorithm as implemented in the open source tool Graphviz, and further builds on it to address Simulink-specific layout requirements, and to automate some useful additional transformations (e.g., splitting a subsystem into two subsystems, transformation of a signal line into a Goto/From connection, etc.). As opposed to other tools described in this paper, this tool is still at the proof-of-concept stage.

The main contribution of this work is a set of tools to be used to aid adoption of some wellknown software engineering principles when designing with Simulink. All the tools have been successfully used on large industrial automotive models: the models contained on average 800 blocks, with hierarchy depth of around six. However, smaller examples have been chosen in this paper to illustrate the tools' capabilities, due to proprietary nature of the industrial models, and for simplicity of exposition. We plan to release the tools in the near future through MATLAB Central, under open source licenses. The tools are currently available for download from http://www.cas.mcmaster.ca/ pantelv/SimulinkTools.

The outline of this paper is as follows. The Signature Tool, the Reach/Coreach Tool, the Data Store Push-Down Tool, and the Auto Layout Tool are presented in Sections 2, 3, 4, and 5, respectively. For each of the tools, we explain and illustrate its main capabilities, implementation, foreseen usages in modelbased design with Simulink, and possible extensions. We also present the execution times of the tools when run on the industrial automotive models. Tools were run on Windows 7, Intel i3-2120 @ 3.30 GHz, 8.0 GB RAM machine. Section 6 concludes the paper, with avenues for future work.

\section{THE SIGNATURE TOOL}

The Signature Tool extracts the signature of a Simulink subsystem. The concept of signatures was first introduced in (Bender et al., 2014a). A signature represents the interface of a Simulink subsystem, making the data flow into and out of the subsystem explicit.

In this section, we first present the background needed to understand signatures and the capabilities of the Signature Tool (Section 2.1). Then, in Sec- 


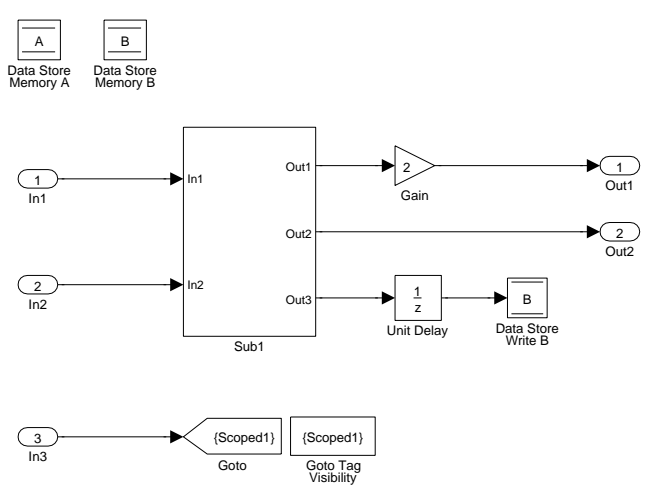

Figure 1: A Simulink model.

tion 2.2, the tool is discussed in details.

\subsection{Analysis of Data Flow in Simulink}

The notion of subsystem is used in Simulink to represent systems inside systems in order to provide for hierarchical modeling. A Simulink subsystem has inports and outports - explicit links to and from the subsystem, respectively. We view inports and outports as the explicit interface of the subsystem. However, there are hidden data dependencies in the Simulink's subsystem: we will refer to those as the subsystem's implicit interface. Hidden dependencies stem from two particular Simulink data mechanisms: data stores and Goto/From blocks:

\subsubsection{Data Store Data Flows}

Data stores are used in Simulink as memory, and are analogous to variables in traditional programming languages. Data stores allow subsystems and referenced models ${ }^{2}$ to share data without having to use inports and outports to pass the data from subsystem to subsystem, or level to level. A data store can be defined in Simulink using a Data Store Memory block. The data store is then referenced using Data Store Read blocks (for reading from the data store) or Data Store Write blocks (for writing into the data store). The scope of a data store is the subsystem where the Data Store Memory block is located, and all the subsystems below it in the model hierarchy, excluding referenced models.

In Figure 1, data store B is defined using Data Store Memory B block. The scope of this data store is the top level of the model and subsystem Sub1. The data store is accessed using Data Store Write B at the same level where it is defined, and it is read from using Data Store Read B at subsystem Sub1.

\footnotetext{
${ }^{2}$ Referenced model is a model referenced from another model using Model block.
}

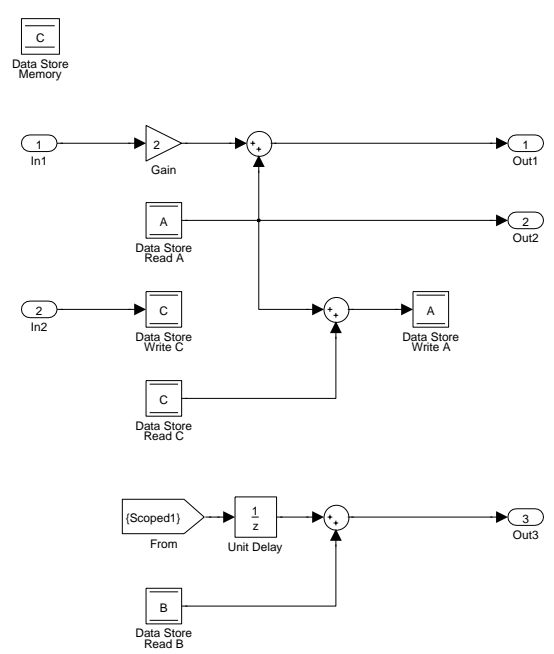

Figure 2: Subsystem Sub1 from Figure 1.

Also, a data store can be implemented in the base workspace using a signal object: it is then called a global data store - it can be accessed from anywhere in the model, including referenced models. When a data store is located higher in the hierarchy than the current subsystem, it will be referred to as an inherited data store for the subsystem. It should be noted, while signatures have previously been defined only for virtual subsystems (Bender et al., 2014a), the current implementation of the Signature Tool is extended to support the extraction of signatures of nonvirtual subsystems as well. Nonvirtual subsystems are subsystems executed as a single unit (atomic execution). A virtual subsystem, on the other hand, is flattened in order to derive the block update order with Simulink ignoring the subsystem's boundaries when determining the order - the subsystem's boundaries do not impact model's behaviour.

\subsubsection{Goto/From Data Flows}

Another mechanism for implicit data flow in Simulink is the Goto/From mechanism. The data fed into a Goto block is passed to its corresponding From blocks (the From blocks with the same $\mathrm{tag}$ ), without a signal line between them. Goto/From blocks are used to implicitly connect blocks, simplifying visual presentation of models. A single Goto block may have multiple From blocks, but a From block may only receive data from a single Goto block. The scope of the Goto block is determined by Goto block's Tag Visibility parameter which can take on the following values:

- Local: Goto and From blocks with the same tag are in the same subsystem.

- Scoped: The scope of the Goto block is deter- 
mined by the position of the corresponding Goto Tag Visibility block: the Goto block and its From blocks have to be in the same subsystem as the Goto Tag Visibility block or lower in the model hierarchy while not crossing a nonvirtual subsystem boundary, i.e., the boundary of an atomic, conditionally executed or function-call subsystem, or a model reference. In Figure 1, scoped tag Scoped1 is defined using Goto Tag Visibility block. This block affects the scope of the Goto block Scoped1 to its immediate left found at the same level, while the corresponding From is found in Sub1, as shown in Figure 2.

- Global: Goto and From blocks with the same tag can be anywhere in the model except in locations that span nonvirtual subsystem boundaries.

In the sequel, we will view global From/Gotos and global data stores as a special case of scoped From/Gotos and data stores, since global tags can be replaced by scoped tags, with a corresponding visibility tag placed in the top-level subsystem, whereas global data stores can be replaced by normal data stores, with the corresponding declaration moved to the top-level subsystem.

\subsection{The Tool}

The signature identifies the parts of the interface of a given Simulink subsystem:

- Explicit interface: Inports and outports,

- Implicit interface: Inherited data stores, and scoped tags defined higher up in the model hierarchy,

- Imposed interface: Data stores and scoped tags defined in the subsystem.

The Signature Tool identifies two useful signatures for a subsystem: strong signature and weak signature. The strong signature identifies the data mechanisms that are accessed by the subsystem or any of its children. The weak signature identifies the data mechanisms that a subsystem can access (those which are declared higher up in the hierarchy), but is not necessarily using.

\subsubsection{Strong and Weak Signatures}

The strong signature of a subsystem contains:

\section{- Inputs:}

- Inports

- Implicit Inputs:

* All the inherited data stores that are only read from in the subsystem or in any of its children
* Scoped tags with Goto Tag Visibility defined higher in the model hierarchy, that have the corresponding From block located in the subsystem or in any of its children, unless the Goto block is also in the subsystem or any of its children. The rationale for excluding tags with both From and Goto blocks from the strong signature is that when the Goto block is located in a subsystem or below it in the hierarchy, it is considered local, since no other subsystem can write into it.

- Outputs:

- Outports

- Implicit Outputs:

* All the inherited data stores that are only written to in the subsystem or in any of its children

* Scoped tags with Goto Tag Visibility located higher in the model hierarchy, that have the corresponding Goto block located in the subsystem or any of its children

- Updates: All the inherited data stores that are both read from and written to in the subsystem or any of its children

- Declarations: All the data stores declared in the subsystem, and scoped tags located in the subsystem

A Simulink model and the contents of its subsystem Sub1 are shown in Figure 1 and Figure 2, respectively. The strong signature for subsystem Sub1 as generated by the Signature Tool is given in Figure 3. The strong signature of Sub1 specifies the data mechanisms that the subsystem or its children access. The subsystem Sub1 both reads from and writes to the inherited data store A - hence, the data store is included in the Updates set of the signature. Sub1, however, only reads from the data store B - consequently, the data store is in the Inputs set of the signature. The subsystem only reads from Scoped 1 tag: that is why the tag belongs to the signature's Inputs.

The weak signature is about a subsystem's context — identifying the data mechanisms the subsystem has access to, but is not necessarily using:

- Inputs:

- Inports

- Implicit Inputs:

* For virtual subsystems, scoped tags declared higher in the hierarchy with the corresponding Goto declared higher in the hierarchy

* For nonvirtual subsystems, this set is empty since From and Goto blocks cannot cross boundaries of non-virtual subsystems 
- Outputs:

- Outports

- Updates:

- Inherited data stores

- Scoped tags located higher in the model hierarchy that do not have corresponding Goto declared higher in the hierarchy

- Declarations: All the data stores declared in the subsystem and scoped tags located in the subsystem

The weak signature of Sub1 from Figure 2 (as the subsystem of system in Figure 1), as extracted by the Signature Tool, is given in Figure 4. Since the weak signature is about the resources that are at Sub1's disposal, all inherited data stores (A and B) are in $U p$ dates since they can be both read from and written to. Sub1 cannot write into Scoped1 since the root level model is already writing into $i^{3}$ - that is why the tag is in the signature's Inputs.

The Signature Tool can extract a subsystem's signature (strong or weak), and then either include it in the subsystem itself, or export it to external documentation. The tool supports virtual and nonvirtual subsystems. The data items in the signature generated by the Signature Tool are presented in the following way (see Figure 3 and Figure 4) for the case when the signatures are included in the subsystem: Data Store Read blocks and Data Store Write blocks and scoped Froms are fed into terminators; all input ports are fed into local Gotos, and output ports are fed from local Froms (our industrial automotive partner uses this technique whenever a port needs to be used multiple times in a model). It is argued in (Bender et al., 2014a) that the behaviour of the subsystem does not change when the signature is included in it.

\subsubsection{Implementation and Applications}

The Signature Tool is implemented using MATLAB functions executed from the command line. The weak signature is implemented as a recursive top-down algorithm on the system tree of a model, while the strong signature is implemented by a bottom-up recursive algorithm. The tool has been used on large industrial automotive Simulink models each implementing a vehicle function: the signature extraction function runtime was below five minutes for each model.

The benefits and applications of signatures are numerous. Signatures represent a simplified view of

\footnotetext{
${ }^{3}$ There cannot be two Goto blocks for the same tag.
}

data flow in and out of the subsystem explicitly identifying hidden dependencies in Simulink models, allowing for easier comprehension of models. Also, the Signature Tool has the capability of extracting strong signatures into external documentation: the generated documentation effectively represents interface specification of a subsystem as a part of the subsystem's software design description documentation. Therefore, this capability automates part of the process of software documentation production: this is exactly how our industrial partner uses the tool.

However, we believe that the future use of the tool, appropriately extended where needed, can go far beyond a simple comprehension aid and documentation generator:

- Signatures can be used to instil software engineering discipline in design with Simulink. For example, the actual interface of a subsystem can be refined from its weak signature generated by the tool: e.g., a data store should be removed from the signature's Updates and included in Inputs if it is to be read-only by the subsystem. The signatures therefore enable information hiding and encapsulation within a Simulink model. Further, if a (interface) specification of a subsystem is given as its signature, and if its weak signature (as extracted by the Signature Tool) has mechanisms not contained in the specification, this is an indication of the subsystem's potential to access data flow mechanisms that may cause unintended interference with other subsystems in the model hierarchy. In fact, a metric based on signatures has been defined in (Bender et al., 2014b) that measures the difference in the number of mechanisms in the subsystem's weak and strong signature: the larger values in the metric indicate potential problematic design as the subsystem has access to far more resources than it is actually using. The tool supports the calculation of the metric.

- A lack of proper consideration of implicit data flow in Simulink testing tools was first noted in (Bender et al., 2014a). Exisiting testing tools typically neglect to account for data flow via data stores when generating a test harness for a subsystem. The subsystem's strong signature extracted by the Signature Tool can be used to generate a test harness that properly accounts for all the incoming/outgoing signals, with the additional benefit of the harness being easily detachable.

- Signatures can be used to classify dynamic inputs (inputs that often change through a simulation run) vs. static (inputs that rarely change through a simulation run). Since signatures make scoped tags as explicit (visible) as inports, applying the 

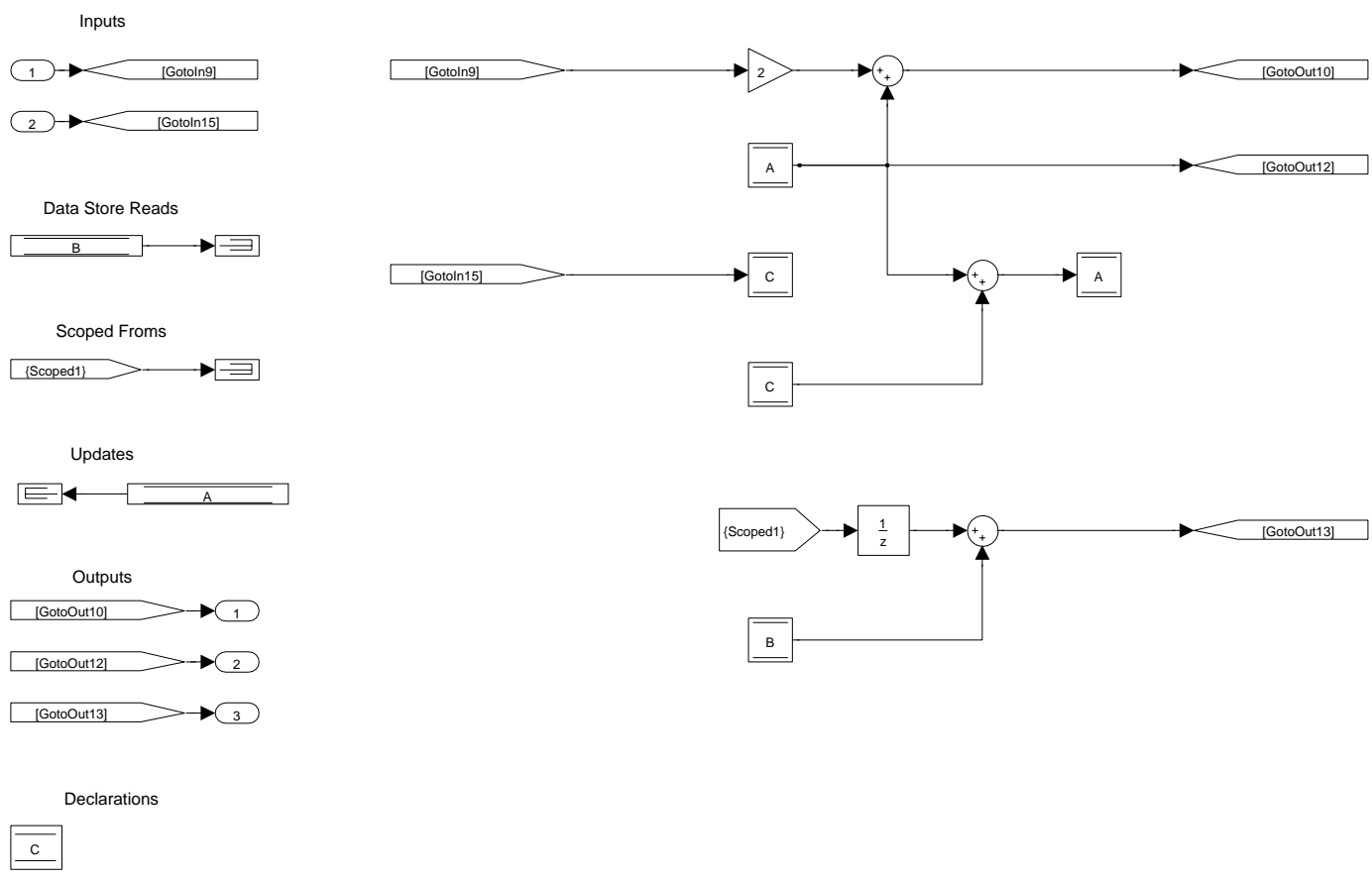

Figure 3: Subsystem Sub1 with included strong signature as generated by the Signature Tool.
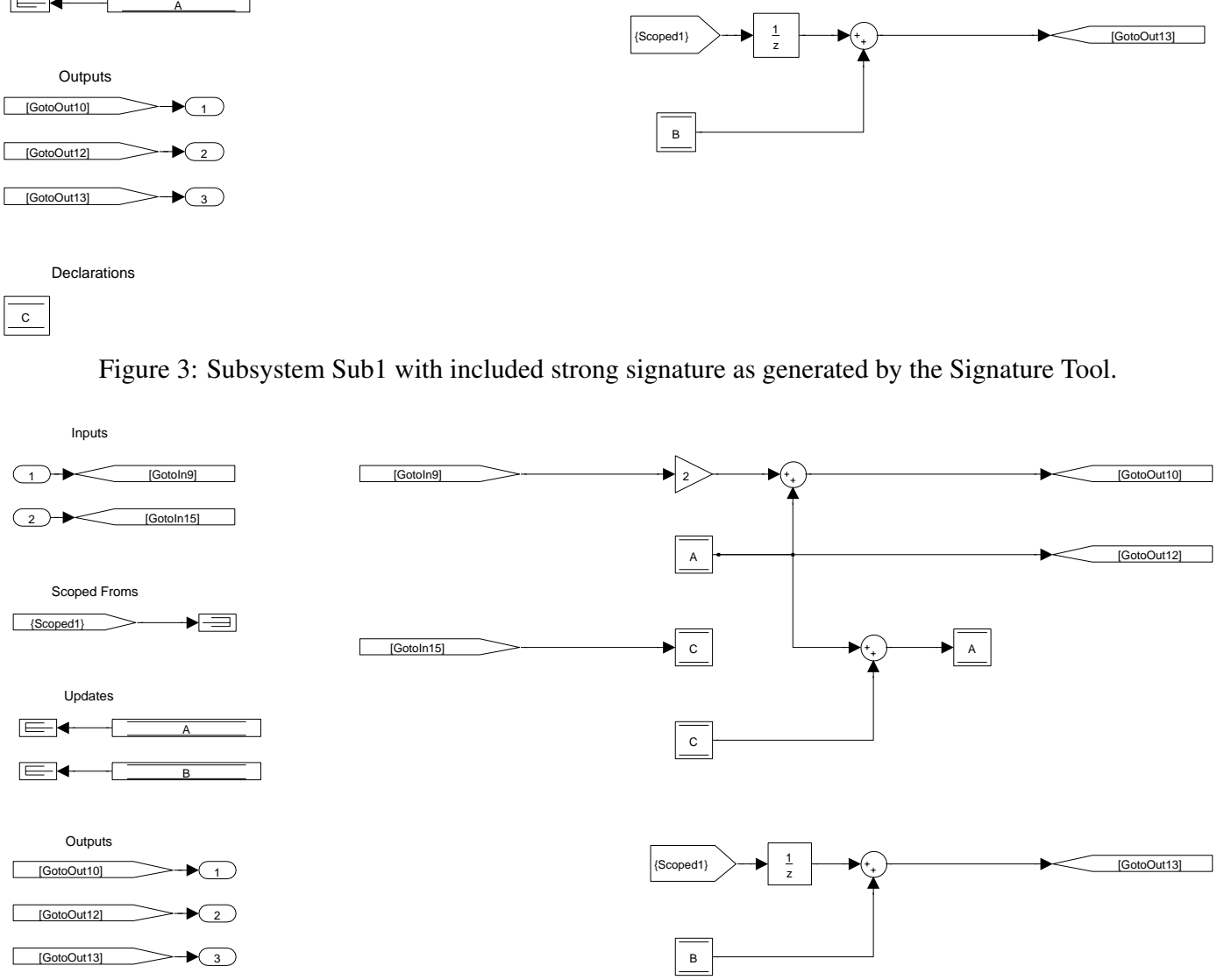

$\bar{c}$

Declarations

Figure 4: Subsystem Sub1 with included weak signature as generated by the Signature Tool.

discipline of e.g., using scoped Goto/Froms for static inputs and using inports for dynamic inputs, would significantly declutter the explicit interface of the subsystem.

- When the subsystem signatures are included in the model, they can be used to incorporate strong typing into subsystems' interfaces. For more details, an interested reader is referred to (Bender et al., 2014a).

\section{THE REACH/COREACH TOOL}

In this section, we present the Reach/Coreach Tool. First, basic capabilities of the tool are introduced. Then, its implementation and applications are ex- 
plained.

\subsection{Tool's Capabilities}

The Reach/Coreach Tool identifies dependencies in a Simulink model in two different ways:

- Reach: For a specified set of blocks (e.g., a set of inports), the tool identifies parts of the model that depend on the specified blocks. The reachable model (the submodel of the original model that consists of the identified blocks, and signals that connect them) is then highlighted.

- Coreach: For a specified set of blocks (e.g., a set of outports), the tool identifies parts of the model that the specified blocks depend on. The coreachable model (the submodel of the original model that consists of the identified blocks, and signals that connect them) is then highlighted.

Once the Reach/Coreach submodel is identified and marked in the Simulink model, the unmarked parts of the model can be trimmed away: the remaining submodel represents a model slice.

The application of the tool on a simple example is illustrated in Figures 5, 6, and 7. The coreachability analysis for outport Out1 results in the submodel marked in blue/yellow colours as shown in Figure 5 (with subsystem WhileSub from Figure 5 shown in Figure 6). The reachability analysis done on inports In 1, In2, and In3 would result in the same submodel being highlighted. The reachability analysis for Data Store Write A in Figure 5 is highlighted in red/black, with the subsystem Sub1 shown in Figure 7. Both reachability and coreachability analysis preserve the hierarchical structure of the model.

The Reach/Coreach Tool tracks and highlights both data flow (data dependencies) and control flow (control dependencies) in the model:

Data Flow. Data flow in Simulink is explained in more detail in Section 2. The Reach/Coreach Tool tracks the explicit data dependencies - the data flow through signal lines. Assuming that changes to an input propagate to changes in any output for any block (except a subsystem), that is, any input can influence any output of a block that is not a subsystem, and by deducing the input-output influence relation of a subsystem from the structure of its components, it is possible to construct the signal line dependency of the model. However, as elaborated in Section 2, there are hidden data dependencies in Simulink: the implicit data flow through data stores and Goto/From blocks. The Reach/Coreach tool tracks these data dependencies as well. For example, in Figure 5, the flow from the Data Store Write A block is tracked to Out2: this comes from the fact that the data store is being read in Sub1. Therefore, the Reach/Coreach Tool accounts for implicit data flow in a model: the tool tracks not only the data flow through inports/outports, but also the data flow via data stores, and Goto/From blocks. This is a major difference between our tool and that of (Reicherdt and Glesner, 2012): their definition of data dependency in Simulink does not account for the implicit data flow. Also, it should be noted that the Reach/Coreach Tool provides for fine-grained tracking of the data flow through Simulink buses.

Control Flow. Control flow logic in Simulink can be implemented using If block and If Action subsystems (for if-else logic), Switch Case block and Switch Action subsystems (for switch logic), and While Iterator and For Iterator subsystems (for loops). For example, the outputs of If block are used to trigger If Action subsystems, depending on the evaluation of a specified condition on inputs of the If block. Action subsystems have an Action Port block: when the input at the port evaluates to true, the subsystem executes. Inputs of If block (Switch Case block) are reached to all the outputs of the block, all the Action subsystems and their outputs. If coreachability analysis is performed from the block whose input is the output of an Action subsystem, it will trace back to Action Port, as well as the data input(s) of the Action subsystem determined by coreachability analysis inside the subsystem; Action Port will be further traced back to all the inputs of the If (Switch Case) block. Note that this is, in general, an overapproximation of actual dependency: an output of If (Switch Case) block can depend only on one of the block's inputs. When it comes to an Iterator subsystem, each block in the subsystem is (control) dependent on the Iterator block in the subsystem, and, therefore, on its inputs. For example, in Figure 5, Out1 is dependent on all the inputs of WhileSub subsystem, since the While Iterator (Figure 6) executes contents of the subsystem based on IC (initial condition) and cond inputs. ${ }^{4}$

Conditional subsystems Enabled Subsystem, Triggered Subsystem, Enabled and Triggered Subsystem, and Function-Call subsystems have a control input in addition to data inputs: when this input satisfies a condition, the corresponding subsystem is executed. For each of those subsystems, outputs are dependent on control inputs: when coreachability analysis is done for one of these subsystems, it traverses through its

\footnotetext{
${ }^{4}$ At the beginning of a time step, if the IC input does not hold, the subsystem is not executed in that time step. If the IC input does hold, the subsystem gets executed, and then, if the cond input is true, the iterator executes the subsystem again. The iterations continues while cond input is true and the number of iterations is less than or equal to the Maximum number of iterations.
} 

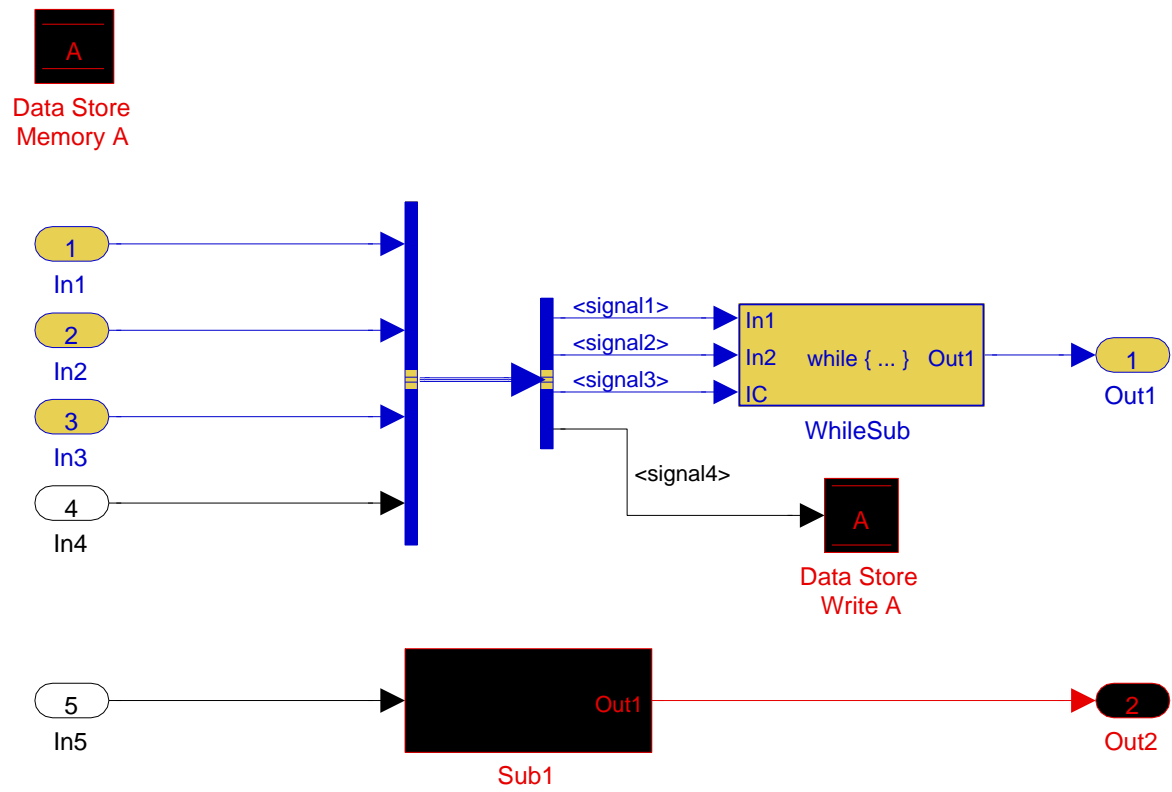

Figure 5: Illustration of Analysis with the Reach/Coreach Tool: Reach for Data Store Write A (red/black), Coreach for Out1 (blue/yellow); Subsystems WhileSub and Sub1 are shown in Figures 6 and 7, respectively.

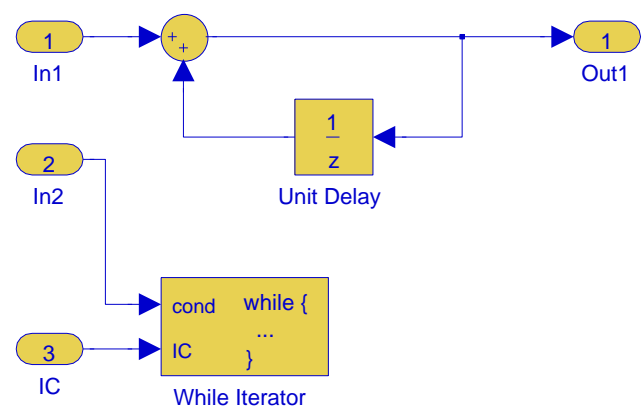

Figure 6: Subsystem WhileSub from Figure 5.

control input. Similarly, if reachability analysis traverses to the control input of a conditional subsystem, it is further propagated to all of the subsystem's outputs. Of course, the dependency of outputs on data inputs is determined by the Reach/Coreach analysis inside the subsystem.

\subsection{Implementation and Applications}

The Reach/Coreach Tool is implemented using MATLAB's object-oriented programming facilities. The Reach and Coreach algorithms are fixed-point algorithms, that identify the immediate reached/coreached blocks of a current set of blocks, starting from the initial specified set of block on which the Reach/Coreach analysis is to be performed. The analyses times do not exceed two seconds for any of the industrial models that were used.

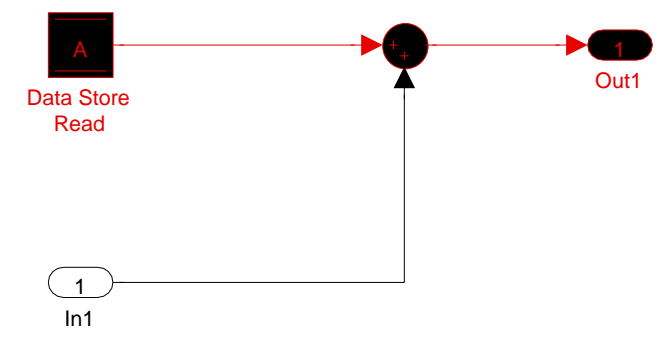

Figure 7: Subsystem Sub1 from Figure 5.

Next, we elaborate on how the tool can be used in a model-based development process.

Refactoring. The tool can be used to find independent/weakly dependent data flows - these might be candidates to be separated into different subsystems.

Comprehension. The Reach/Coreach Tool depicts the dependencies in a Simulink model, effectively representing its dependency diagram. While the signatures address the comprehension issue at the level of a subsystem, the Reach/Coreach Tool offers an abstracted view of data and control flow from given blocks backwards to inputs, or forwards to outputs. Both the views generated by the Signature Tool, and the views generated by Reach/Coreach Tool, are data flow views - and, as claimed in (Quante, 2013): "such views can be very helpful for tracking data flows through a system - especially when there 


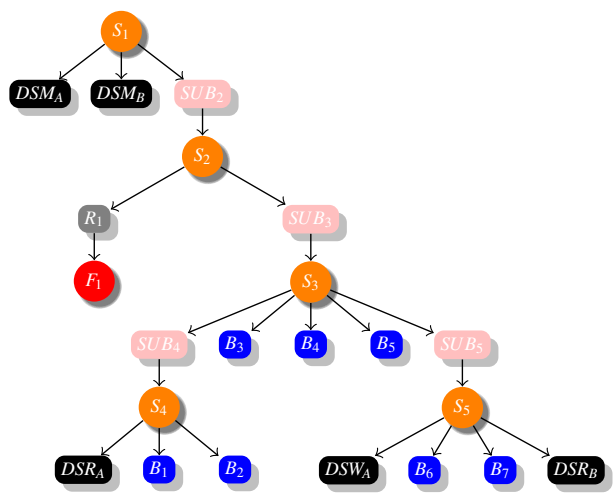

Figure 8: Data store push-down: before.

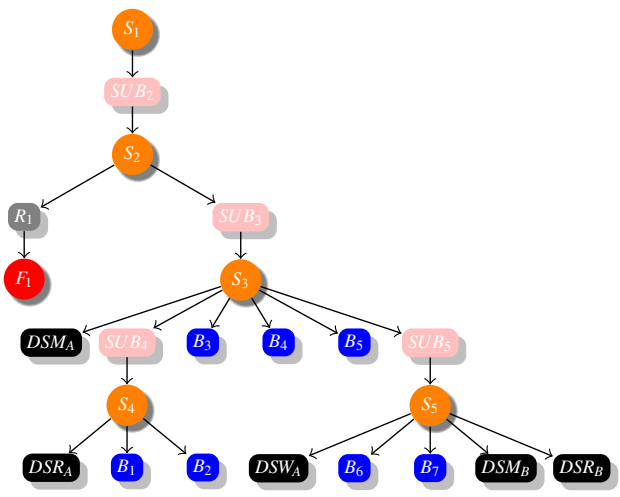

Figure 9: Data store push-down: after. are additional hidden dependencies." Further, by trimming the extraneous blocks from a model, it is possible for a user to gain a greater understanding of the structure of the model. For review purposes, the Reach/Coreach analyses can assist reviewers in fully grasping data and control dependencies in a model: understanding the flow in complex models is very hard without proper abstractions even when the models are well-documented.

Dead Code. The tool can be used to find unreachable parts of a model: once the reachability analysis is performed on all of model's inputs (inports), non-marked blocks/signals represent unreachable parts of the model. When the coreachability analysis is performed on all of model's outputs (outports), the extraneous blocks are unnecessary in the sense that they have no (data or control) effect on the outputs of the model.

Impact Analysis. Since the tool identifies the parts of a model affected by a change of a given block, it provides for impact analysis. The impact analysis can be of great value in indicating what effect a change in requirements can have on system's design. During both initial design and refactoring, the tool can be used for preliminary evaluation of different designs with respect to the extent that the future anticipated requirements changes will have on the system's design. Therefore, the tool would help the developer design for change. Further, impact analysis can be extremely beneficial in alleviating verification efforts, which are typically very large, especially in the case of safety-critical systems. Verification efforts can be decreased by focusing on the parts of the system that the outputs (or, more generally, the data items) of interest depend on: we view the Reach/Coreach Tool as a useful means of providing impact analysis that avoids the costly analysis of the parts of the system that are not affected by a change.

\section{THE DATA STORE PUSH-DOWN TOOL}

We have noticed that some of the industrial models we have been working with define most of their data stores at the top level of a model's hierarchy. This practice is analogous to programming using a large number of global variables, and it is considered a bad software engineering practice.

Data stores, like variables in traditional programming languages, should be properly scoped in order to disable inadvertent/unwanted access to the data stores. Also, the proper scoping declutters the interface of a subsystem by hiding low-level details of the subsystem, therefore providing proper encapsulation. Proper data store scoping also reduces the number of (implicit) inputs for testing, resulting in possibly fewer generated tests (test steps). Therefore, proper scoping of data stores enhances comprehensibility, maintainability, testability, and reusability of Simulink subsystems.

In this section, the concept of the data store pushdown operation is discussed first. Then, tool's implementation and applications are detailed.

\subsection{Illustration of Data Store Push-down Operation}

We have already discussed scope associated with data stores and Goto/From blocks. A block falls within the scope of another block $b$ if it is contained in a system that belongs to the scope of the block $b$. Our goal is to limit the scope of data stores as much as possible. For example, if a Data Store Memory occurs high in the model hierarchy, and there are two data store references (Data Store Read or Data Store Write blocks) lower in the hierarchy, the goal is to push-down the data store (Data Store Memory block) to the smallest 


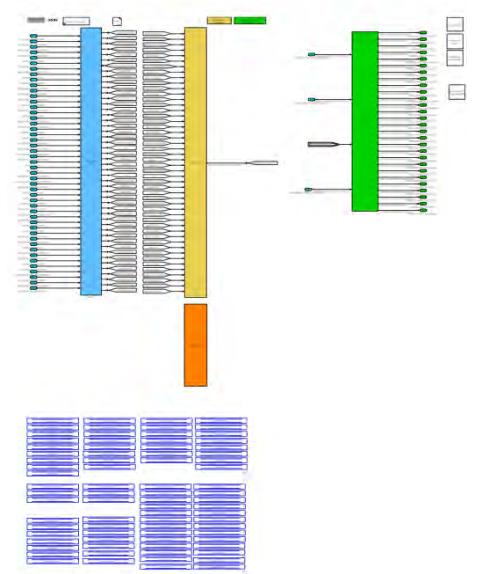

Figure 10: The top level of the industrial model before the push-down operation.

subsystem such that both references are still within the scope of the data store.

In Figure 8, a system is represented before the data store push-down operation has been applied to it. The orange nodes represent systems, the rectangular elements are blocks, the pink blocks are subsystem blocks. Grey blocks are reference blocks (blocks that refer to a block in another file, here denoted by a red node). Arrows emanating from systems to blocks denote membership of the block in the system. An arrow emanating from a subsystem block to system denotes that the system is associated with the subsystem; similar relationship exists for references and files. Figure 9 shows the system after the data store push-down operation has been applied to it.

\subsection{Implementation and Application}

The push-down algorithm is implemented as a single iterative MATLAB function. Firstly, the push-down algorithm searches the model for all Data Store Memory blocks. Then, it searches for all the corresponding Data Store Read and Data Store Write blocks. The addresses of these corresponding Data Store Read and Data Store Write blocks are then parsed to find their lowest common ancestor. Lastly, unless the lowest common ancestors reside in a library linked subsystem, the Data Store Memory blocks are then pushed to their respective lowest common ancestors. Execution times of the algorithm on the large industrial models (containing many Data Store Memory blocks at the top level (around 50) and deep model hierarchy (of depth 6)) do not exceed one second.

Given its importance, we propose proper scoping of variables to be included in the modeling style guidelines for Simulink. With Simulink/Stateflow

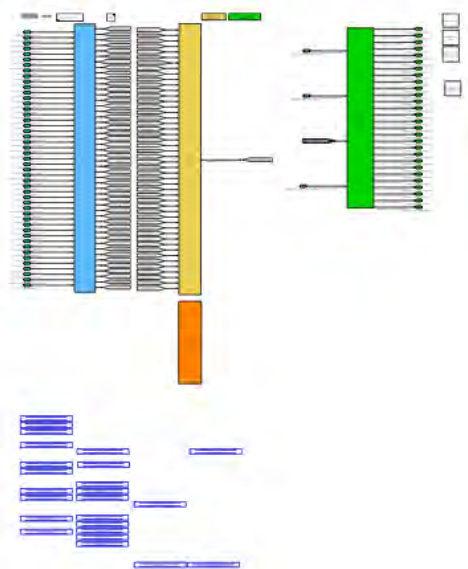

Figure 11: The top level of the industrial model after the push-down operation.

emerging as a leading environment for model-based design of embedded systems, a number of guidelines have been created to assist designers in modeling. Guidelines typically provide a wide range of rules/recommendations (e.g., naming conventions, usage of Simulink patterns for different constructs (e.g., case constructs), grouping of blocks into subsystems, etc.). In the automotive industry, the most notable modeling standard is published by The MathWorks Automotive Advisory Board (MAAB) (MathWorks, 2014b). In addition, companies use in-house guidelines to improve the quality of their software. Inhouse guidelines typically contain a number of checks from standard guidelines. Adherence to the rules improves software testability, understandability, and maintainability. Also, the compliance enhances simulation and code generation capabilities. For example, each of the MAAB rules/recommendations for Simulink/Stateflow is justified by one or more of the following:

- Easily understood algorithms (readable models, uniform appearance of models, code, and documentation, clean interfaces, professional documentation),

- Effective development process and workflow (ease of maintenance, rapid model changes, reusable components, etc.),

- Efficient simulation and analysis,

- Generation of code that is efficient and effective for embedded systems,

- Ability to verify and validate a model and generated code (requirements traceability, testing, problem-free system integration, clean interfaces).

In order to include proper scoping of data stores in modeling guidelines, a rule can be formulated to re- 


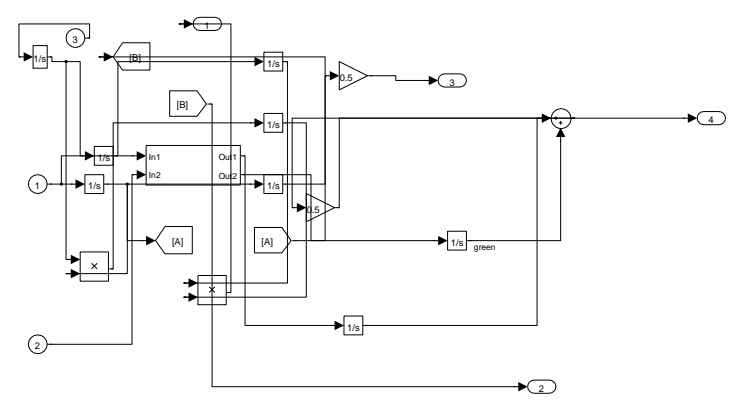

Figure 12: Simulink Model: Before Layout.

quire that each data store (with certain exceptions) be defined at the lowest hierarchy level such that all the references to the data store are still within its scope. The exceptions account for situations where a developer might desire to leave a data store defined at a higher hierarchy level than currently needed foreseeing that the data store will be used in future by other subsystem(s) at the same or higher hierarchy levels. Therefore, the user of the tool should be able to choose the data stores that should not be pushed down the hierarchy. Our tool supports this feature. A straightforward modification of our tool can then be used to check for the compliance of a Simulink model with the rule, and then repair the model (perform the push-down operation) if needed so that the model adheres to the rule.

An interesting synergy between signatures and the push-down operation was demonstrated in (Bender et al., 2014b). The push-down operation was applied on an industrial automotive model shown in Figure 10. The number of data stores at the root level (data stores are represented by blue rectangles at the bottom of the figure) has been significantly decreased by the push-down operation as illustrated by Figure 11 (not all the details in models in both figures are legible for confidentiality purposes). From a software engineering perspective, the push-down operation clears the interfaces of model's subsystems at different hierarchy levels: the impact of the pushdown operation is the largest for the subsystems at the model's root level. This change in subsystems' interfaces is obvious with simple visual inspection of their signatures as generated by the Signature Tool. Further, the Signature Tool was used on the model's subsystems to calculate the difference in the number of data items in a subsystem's weak and strong signature: this metric as formalized in (Bender et al., 2014b) and, as explained in Section 2, indicates the quality of modularization of designs in Simulink. The values of the metric for model's subsystems, before and after the push-down operation, indicate the significant improvement in modularity realized by the push-down operation.

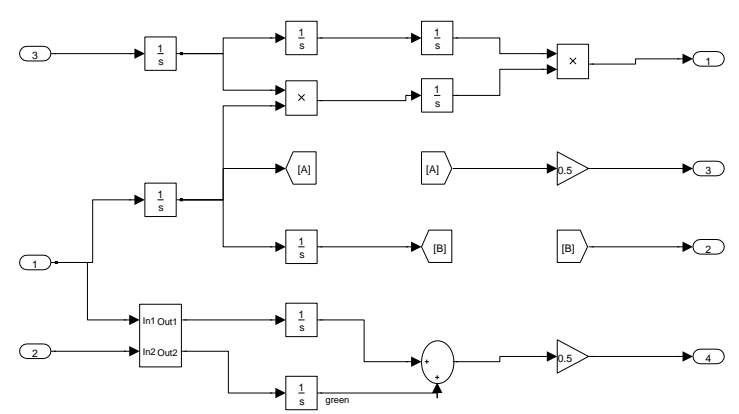

Figure 13: Simulink Model: After Layout.

\section{THE AUTO LAYOUT TOOL}

In a modeling environment such as Simulink, the readability of models is largely determined by graphical layout of blocks and lines in the model. Since most refactoring operations perturb model layout and manual readjustment of model layout is a tedious and error-prone process if performed manually, automatic layout is viewed as an essential component of model refactoring. However, there does not exist a commercial tool that comprehensively tackles the automatic layout of Simulink models. There are a number of commercial tools that check Simulink models for compliance with modeling style guidelines (MXAM by Model Engineering Solutions (Model Engineering Solutions, 2014), Simulink Model Advisor by the MathWorks (MathWorks, 2014a) with the Verification and Validation Toolbox (MathWorks, 2014c), etc.). These tools also offer automatic repair for some of the rules once a model fails a rule; however, the repair capabilities of these tools are modest at best. While a number of layout algorithms have been proposed (e.g., (Klauske and Dziobek, 2010; Klauske et al., 2012)), to the best of our knowledge, no tools based on these papers are available for download.

Our layout tool uses an already existing graph drawing algorithm (Gansner et al., 1993) implemented in Graphviz. Graphviz is a set of open-source tools for drawing graphs represented by the DOT graph description language. More precisely, our tool harnesses the Graphviz's layout engine dot for the autopositioning of model blocks; lines are then autogenerated using Simulink's built-in automatic line positioning support. Graphviz's tool dot uses the algorithm of (Gansner et al., 1993) to rearrange blocks and lines in a consistent, organized manner to maximize readability (the same algorithm is also partially used by (Klauske and Dziobek, 2010)). The tool resizes blocks based on number of inputs and outputs, and organizes the lines such that the number of crossings is minimized. See Figure 12 for a model before au- 
tomatic layout, and Figure 13 for the model after automatic layout. Note that model comprehension and readability have markedly improved.

As opposed to the tools described in the previous sections, this tool is at the proof-of-concept stage. We are currently refining the layout engine to accommodate Simulink-specific layout requirements (for example, outports should be placed on the right side, unless they are moved to prevent crossings). Further, we are also implementing a number of refactoring transformations that are not natively automatically supported by Simulink: e.g., splitting/merging subsystems.

\section{CONCLUSIONS}

Automated support is an essential part of any software development process. This paper presents a set of tools that help automate the application of some traditional software engineering practices when designing with Simulink. While the paper illustrates the use of the tools on simple toy examples, the tools have been proven on large, industrial models from the automotive industry, that could not be presented in the paper due to their proprietary nature. The concepts presented in this paper, and tools based on the concepts, represent the beginning of our investigation of the issues of integrating some traditional software engineering practices in design with Simulink.

\section{REFERENCES}

Bender, M., Laurin, K., Lawford, M., Ong, J., Postma, S., and Pantelic, V. (2014a). Signature required: Making Simulink data flow and interfaces explicit. In Proceedings of 2nd International Conference on Model-Driven Engineering and Software Development (MODELSWARD 2014), pages 119-131. SCITEPRESS.

Bender, M., Laurin, K., Lawford, M., Pantelic, V., Korobkine, A., Ong, J., Mackenzie, B., Bialy, M., and Postma, S. (2014b). Signature required: Making Simulink data flow and interfaces explicit. In Science of Computer Programming, Special issue on ModelDriven Development. Submitted in June.

Gansner, E. R., Koutsofios, E., North, S. C., and Vo, K.-P. (1993). A technique for drawing directed graphs. Software Engineering, IEEE Transactions on, 19(3):214230.

Klauske, L., Schulze, C., Spnemann, M., and von Hanxleden, R. (2012). Improved layout for data flow diagrams with port constraints. In Cox, P., Plimmer, B., and Rodgers, P., editors, Diagrammatic Representation and Inference, volume 7352 of Lecture Notes in
Computer Science, pages 65-79. Springer Berlin Heidelberg.

Klauske, L. K. and Dziobek, C. (2010). Improving modeling usability: Automated layout generation for simulink. In Proceedings of the MathWorks Automotive Conference, MAC.

MathWorks, T. (2014a). Model Advisor. http://www. mathworks.com/help/simulink/ug/consulting-themodel-advisor.html. [Online; accessed September 2014].

MathWorks, T. (2014b). The MathWorks Automotive Advisory Board. http://www. mathworks.com/automotive/standards/maab.html. [Online; accessed September 2014].

MathWorks, T. (2014c). Verification and Validation Toolbox. http://www. mathworks.com/products/simverification/. [Online; accessed September 2014].

Model Engineering Solutions (2014). MES Model Examiner (MXAM DRIVE). http://www. modelengineers.com/en/model-examiner.html. [Online; accessed September 2014].

Quante, J. (2013). Views for efficient program understanding of automotive software. Softwaretechnik-Trends, $33(2)$.

Reicherdt, R. and Glesner, S. (2012). Slicing MATLAB Simulink models. In Software Engineering (ICSE), 2012 34th International Conference on, pages 551561. IEEE. 